\title{
Review of tradeoffs for quenched avalanche photodiode sensors for imaging turbid media
}

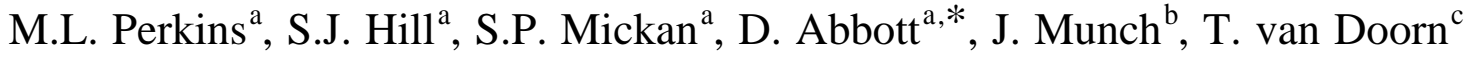 \\ ${ }^{\mathrm{a}}$ Center for Biomedical Engineering (CBME), Electrical and Electronic Engineering Department, University of Adelaide, \\ Adelaide, SA 5005, Australia \\ ${ }^{\mathrm{b}}$ Physics Department, University of Adelaide, Adelaide, SA 5005, Australia \\ ${ }^{\mathrm{c}}$ Royal Adelaide Hospital, Adelaide, SA 5005, Australia
}

Received 15 December 1999; accepted 30 December 1999

\begin{abstract}
We evaluate the efficiency of a new optoelectronic quenched avalanche photodiode sensor (QAPD), with the potential of enabling highresolution imaging through turbid media with femtosecond-lasers. Our target application is for imaging cancer in the human breast.

We aim to improve the contrast ratio of the unscattered, image bearing photons by reducing or removing the large background of multiply scattered photons that result in poor spatial resolution. The approach that we are taking is to use temporal discrimination: ultra-short (less than a picosecond) laser pulses and time gated detection can select only those photons which travel almost straight through the tissue. The feasibility of resolving a $1 \mathrm{~mm}$ structure in $50 \mathrm{~mm}$ turbid tissue sample, using our system, will be discussed-there are no results reported to date that approach this performance.

We aim to enhance the detection of short pulses through time resolved single photon counting technique. In this technique, the earliest arriving photon of the transmitted pulse is detected with a QAPD. When combined with an efficient electronic quenching circuit, the QAPD is insensitive to the later arriving diffuse photons. High accuracy and efficient measurement of the arrival time of the first photons is achieved by time to amplitude conversion electronics with a temporal resolution of $3 \mathrm{ps}$.

This paper contains a discussion of the laser source, detection circuit, including QAPD, high-speed comparator, critical biasing and intensity counter. (C) 2000 Elsevier Science Ltd. All rights reserved.
\end{abstract}

Keywords: Avalanche photodiode; Imaging; Single photon; Quenching; Laser

\section{Introduction}

The crucial components of the laser detection circuit design are the avalanche photodiode, sometimes referred to as an APD, and the quenching circuit. These two parts combined are expressed wholly as a QAPD (quenched avalanche photodiode). The quenching is not actually part of the diode itself, but is an external circuit that can be implemented in a number of ways.

The laser gives out ultra-short pulses of light that are very intense. This light is passed through a turbid media (such as a human breast) where most of the photons are scattered, with a few passing straight through. Our circuit determines those which have passed straight through and, using time gating, can tell us whether or not there was an opaque object

\footnotetext{
* Corresponding author. Tel.: + 61-8-8303-5748; fax: + 61-8-83034360.

E-mail address: dabbott@eleceng.adelaide.edu.au (D. Abbott).
}

in the path. By moving the laser over the surface of the media, we can form an image of what is inside.

Although our target application is for use in the detection of cancer, QAPDs also have other uses including astronomical observation, optical range finding, optical fiber testing and fault location, ultra-sensitive fluorescence, etc. This technology can also be used in the medical context to complement the current mammogram. This method does not involve the use of X-rays and is more suitable to a larger range of patients. It can also detect diffuse tumors, which can be difficult to find using conventional techniques.

Our work is closely related to research into the fields of single photon counting and detection using QAPDs $[1,2,5]$.

\section{Laser source}

Since the 1920s, the use of visible and infrared wavelength radiation has been postulated as an imaging source in preference to the ionizing X-rays with the dangers and 


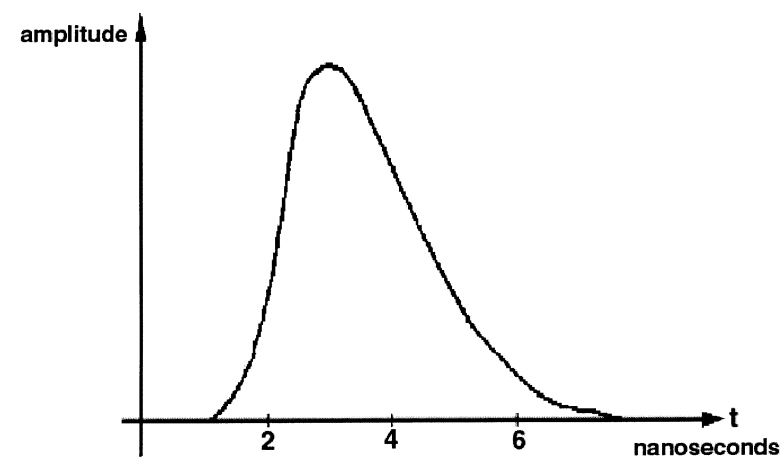

Fig. 1. Femtosecond pulse waveform after passing through medium.

concerns over cancerous developments. With modern advances in light sources (especially femtosecond light sources) and optoelectronic components for the telecommunications industry, interest has been stimulated in the possibility of imaging in these wavelengths. Our chosen laser source for trials is a Ti-Sapphire mode locked laser. The repetition rate will be around $100 \mathrm{MHz}$. The laser will be used to produce a pulse of approximately $10 \times 10^{-15} \mathrm{~s}$, or $10 \mathrm{fs}$ in duration.

The pulse originates as a very clean, sharp edged pulse. It is split for use as a timing reference beam for the time gating applications, and then it is passed into the turbid medium under investigation. In the passage through the medium the leading edge of the femtosecond-pulse is spread due to the interactions between the photons and the turbid medium. Photons are deflected off the shortest, fastest path through the medium and then further deflected in multiple interactions which could see the photons passed out of the medium in a direction such that they will still impact with the detector. The shape of the pulse on leaving the turbid medium is similar to that shown in Fig. 1.

The point of interest for our target applications is the very first rising edge of this waveform. This is where all the image-bearing photons are. This is demonstrated in Fig. 2.

The definitions of the parameters are as follows. Here, $t_{\min }$ is the minimum amount of time required for the photons to travel through the medium by travelling over the shortest path. These are the first arriving photons, and are the ones that have been deflected the least in the passage through the medium. These photons are carrying the image that we wish to retrieve. The parameter $t_{\text {res }}$ is the time required for a set value of resolution. Our aims are to achieve a resolution of $1 \mathrm{~mm}$ in a $50 \mathrm{~mm}$ turbid medium. Therefore the $t_{\text {res }}$ that suits this requirement would be $t_{\text {res }}=1 \mathrm{~mm} / \mathrm{c}=3 \mathrm{ps}$. ( $c=3 \times 10^{8} \mathrm{~m} \mathrm{~s}^{-1}$ — the speed of light). In this time we must detect the arriving photons, and quench the APD to ensure it is insensitive to the later arriving photons as these will reduce the image resolution. It is not important to know which photon in the rising edge we detect, it is however important to ensure that the photon arrived before $t_{\text {res }}$. If the photons arrive after $t_{\text {res }}$ and are detected then the image clarity is reduced.

\section{Avalanche photodiode properties and characteristics}

For low-light and single photon detection in the visible and near IR range there are basically three detector choices. The first is the silicon $\mathrm{P}-\mathrm{I}-\mathrm{N}$ diode, second is the photomultiplier tube (PMT) and the last is the Avalanche Photodiode. The APD has the advantage of a higher quantum efficiency than PMTs, and higher speed and sensitivity than $\mathrm{P}-\mathrm{I}-\mathrm{N}$ diodes.

The selection of the correct avalanche photodiode is crucial to the performance of the circuit [4]. It can be made of silicon for the $300-1100 \mathrm{~nm}$ range, Germanium for $800-1600 \mathrm{~nm}$ range or Indium Gallium-Arsenide (InGaAs) for the 900-1700 $\mathrm{nm}$ range. Ideally, an APD with zero dark current, $100 \%$ detection efficiency, ultrafast response time, low probability of carrier trapping and of course low-cost would be the perfect choice. In reality however, this is impossible to achieve because of the need to trade-off conflicting design requirements.

In the Geiger mode, the APD is biased above its breakdown voltage for operation at very high gain. In this mode, the APD can be used to count individual photon arrivals that will trigger a significant current. However, the APD may also be falsely triggered by the "dark" current resulting in false counts, not caused by a photon but by thermal generation of electron-hole pairs. The value of the bulk dark current is therefore an important consideration when selecting an APD. The value of this dark current can be reduced

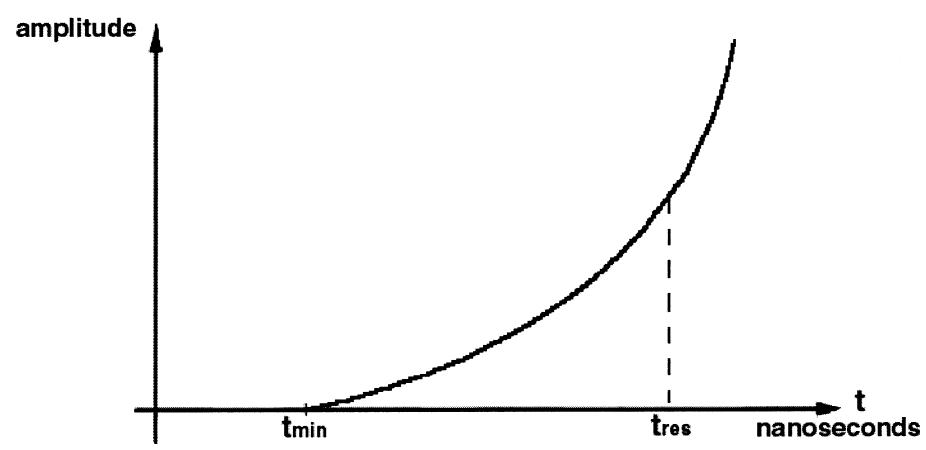

Fig. 2. Relevant component of pulse waveform. 


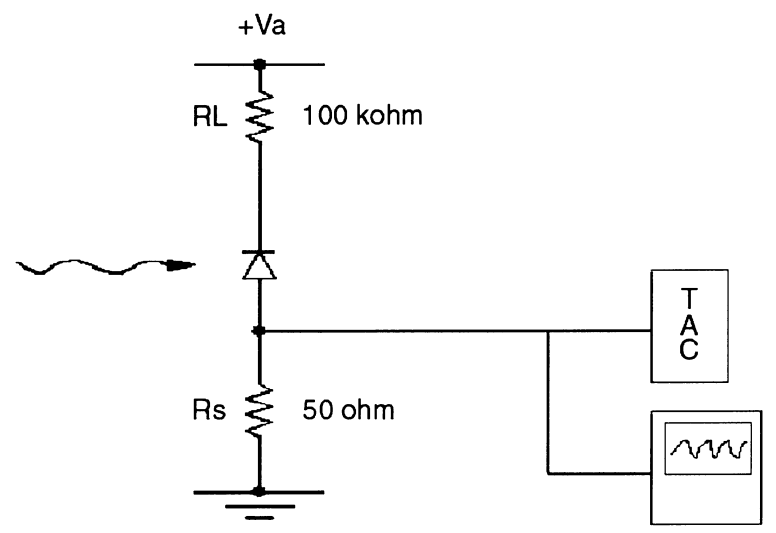

Fig. 3. Diagram of a passive quenching circuit.

exponentially by cooling the diode. Some commercially available diodes [7] use TE single and double stage cooling integrated into the diode package to overcome this problem. This cooling also has the advantage of offering more stable characteristics and a higher reliability and repeatability rate.

Photon detection efficiency relates to the probability of a photoelectron generating an avalanche. This probability increases with bias voltage. The photon detection efficiency in Geiger mode is quite high $(>80 \%)$ once the diode is biased a few volts above the breakdown voltage. Dead time is a phenomenon that occurs for a short time after the detection of a photon. In this time, the APD is insensitive to any later arriving photons which limits the count rate of these diodes. The time between pulses of the source laser is $10 \mathrm{~ns}$ and so the dead time must be less than $10 \mathrm{~ns}$ so that pulses are not missed.

Afterpulsing is another property of the Geiger mode APD that has to be taken into account. During an avalanche, some of the large number of carriers may become trapped inside the depletion layer. If the trapped carriers are released after the diode has been recharged, they can initiate breakdown. This "afterpulse" probability also increases with bias voltage.

When considering APD designs there are two to be considered, which are the thick junction APD and the thin junction APD. The thick junction photodiode works at much higher bias voltages (due to the need to have a dense electric field in the junction), however, it has higher detection efficiencies. The thin junction photodiode operates at lower bias voltages, and has significantly reduced detection efficiency. However the thin junction photodiodes are also reported to switch around one order of magnitude faster than the thick junction photodiodes. In order to obtain the picosecond resolution necessary for our application, a thin-junction, ultra-fast APD is needed. The reason for this is the critical specification of the rise time, and not the detection efficiency.

\section{Quenching circuit}

The critical piece of circuitry with which the designer is faced with is the quenching circuit. The quenching circuit serves a large number of roles, and is a major design challenge. The first task of the quenching circuit is to enable the APD to be biased correctly for normal operation. This means that it must be capable of handling voltages around $50 \mathrm{~V}$ for a thin junction APD, or upwards of 100-300 V for a thick junction APD. After this point, there are two main design formats that can be followed. The first one to be described is passive quenching [6], the second is active quenching.

Passive quenching is much simpler than active quenching, which is a plus for the designer, however this comes with several drawbacks of which the most important are speed and flexibility. The passive quenching circuit is slow for two reasons. The first is the resistance of the quenching circuit and the capacitance of the APD that, combined with other stray capacitances form a large time constant which prevents the bias voltage from dropping rapidly. This means that the quenching action is delayed slightly, which can cause problems with the accuracy of the photon arrival detection and subsequent detections, as well as the possibility of increased power loss and hence temperature rise in the APD.

The second restriction is the possibility of carriers becoming trapped in the junction of the APD. Due to the high voltages and large currents that occur over and in the APD, carriers can become caught in the APD junction. The longer the APD is left unquenched the more carriers that are potentially trapped. Once the bias voltage has dropped and the current reset, these carriers can spontaneously re-emit. If the bias voltage over the APD is reset too early, then these re-emitted carriers can falsely re-trigger the avalanche. To overcome this problem the bias voltage must be held low for a certain amount of time to allow these trapped carriers to be freed without restarting the avalanche. The longer the bias is held low, the lower the probability of re-triggering due to re-emitted carriers.

The passive circuit also lacks a lot of the flexibility of the active circuit. It is very difficult to vary the dead time of the passive quenching circuit due to its dependence on circuit component values. This makes it difficult to ensure the dead time is sufficient to prevent re-triggering, and it also makes it difficult to remove unnecessary delays in reapplying the bias voltage. These factors combined mean that the repetition rate of the passive quenching circuit is low, as well as having a slower detection time. With the strict requirements that we are aiming to satisfy, these make the passive quenching circuit mostly unsuitable. An example of a basic passive quenching circuit can be seen in Fig. 3 .

The second form of quenching is the active quenching circuit, as shown in Fig. 4. As its name implies, the active circuit forces back on the APD to drop the bias voltage much quicker. This enables significant speed improvements over the passive circuit, however it is no simple task to design an effective active circuit. The active circuit can be loosely broken down into further sub-circuits, however 


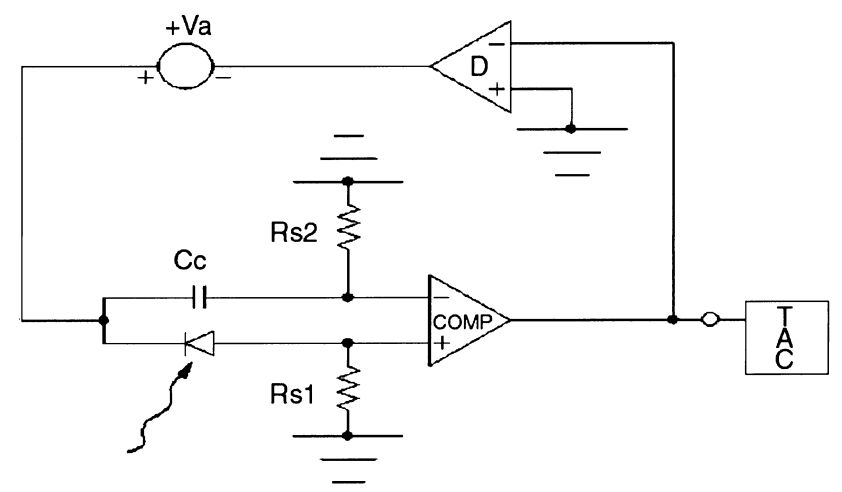

Fig. 4. Simplified active quenching circuit. The component D is the quench/ reset driver.

these sub-circuits are more to aid the understanding of the active circuit operation as opposed to making the design task easier. Due to the time constraints involved there is a significant amount of component integration to reduce propagation delays as well as stray capacitances.

The first task of the active quenching circuit is to act as a comparator. The need for the comparator is twofold. Firstly, it is necessary to discriminate between the approximately constant dark current background that is flowing through the APD due to thermally generated carriers. It must be able to detect the sharply rising avalanche current caused by an incoming photon and avoid causing any false detections caused by any fluctuation in the dark current background. Secondly it must be able to produce a useable signal output which is representative of the current pulse generated by the APD.

The comparator output is used for two tasks. Firstly it provides a reference which can be used by external counting devices to record the number of avalanche events which occur. Secondly it must initiate the voltage pulse which is used to reduce the bias voltage over the APD. Due to this it is necessary to ensure that any propagation delay due to the comparator be minimized.

A voltage driver is required to enable the large voltage quenching pulses (up to $50 \mathrm{~V}$ ) to be generated. This pulse is required to force back onto the APD and reduce the bias voltage such that the avalanche current is quenched, and such that the APD is insensitive to later arriving photons. The reason for the size of the voltage pulse is due to the biasing arrangement that is employed for APDs. They can be biased over $20 \mathrm{~V}$ above the breakdown voltage for fast operation. This excess voltage ensures the switching time, or the rise time of the avalanche current is as small as possible. This voltage pulse can also be used as the reference for the external counting circuitry, however it may be more practical and simpler to use the comparator output. Again it is important to minimize the propagation delay to ensure the required resolution is achieved.

The final component is a timer circuit, which is used to control the dead time of the quenching circuit. The circuitry for this is not as critical to achieving the required resolutions as are the aforementioned issues. If the 3 ps quenching time can be achieved, then the repetition rate of $100 \mathrm{MHz}$ allows plenty of time for the bias to be held low, to reduce the possibility of false avalanches caused due to the re-emission of trapped carriers in the junction. However it is still necessary to ensure that the timer can hold the bias low without adding unnecessary capacitances to the design which may affect the other more critical rise times.

There is also a hybrid technique that can be used to provide the necessary quenching circuitry. A combination of passive and active circuit elements can be used. This enables a simpler design to be used, while still allowing some control over the dead time and the use of a voltage pulse to speed up quenching. A possible design is to use passive quenching for the first stage. An active circuit detects when the passive circuit begins to reduce the APD bias voltage, and then helps by applying a voltage pulse as well.

The active circuit holds the voltage low for a time before re-applying the bias to enable the next detection event to occur. This enables a much simpler design to be used, however the time constant caused by the combination of the APD capacitances and the passive quenching resistances means that the quenching speed is limited. It does however out-perform a straight passive circuit, at the cost of complexity and designer effort. An example of a circuit which has been designed similar to this can be found in "Compact active quenching circuit for fast photon counting with avalanche photodiodes" [3].

Performance limitations are heavily dependent on switching delays, which are directly related to the capacitance of the circuit. One of the major capacitances of the aforementioned circuits is the junction capacitance of the avalanche photodiode. Very fast switching times are limited by this capacitance-which is set by the design process used by the manufacturer. Mounting the components on stripline reduces this capacitance by up to a factor of 10 . This also has the advantage of being able to remotely mount the circuit by means of coupling with co-axial line.

\section{Pulse detection}

In order to achieve our target resolution, the need for a very fast photodiode has been outlined. To measure this event, it follows that a very high speed sampling oscilloscope or a very fast time to digital converter (TDC) must be used. Measuring the number of counts per second of photons that have traveled straight through the media gives us an idea of what is inside-i.e. forms an image. The time-gating component of the circuit discards any counts that occur after the cutoff time $\left(t_{\text {res }}\right)$. This technique is particularly useful in that, unlike X-rays, it can better detect diffuse tumors.

To ensure that no photons arrive after $t_{\text {res }}$, a time gating technique must be implemented. One method of accomplishing this is to split the source beam into two and have 


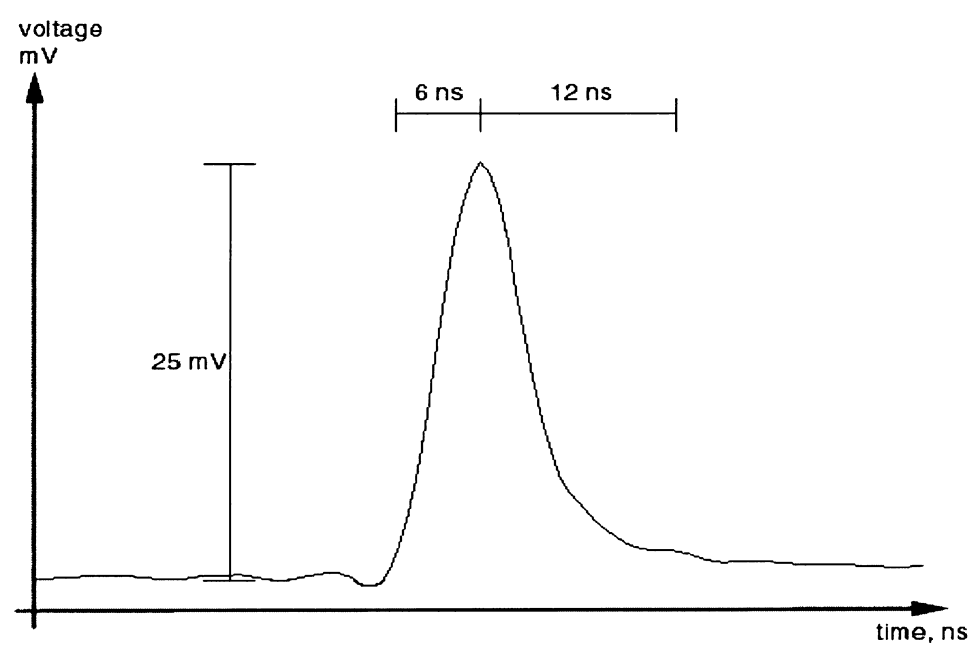

Fig. 5. Output quenched waveform using passive quenching and the C30902E from EG and G.

two detectors. One of the beams passes through the turbid media (beam 1 into detector 1), while the other is uninterrupted (beam 2 into detector 2). If the first detector has not avalanched by $t_{\text {res }}$ after detector 2 has avalanched, then detector 2 reduces the bias voltage of detector 1 such that it is now insensitive to further arriving photons. Another method of time gating involves the use of an electrical signal that coincides with the pulse output of the laser. This signal can be used as a reference to find $t_{\text {res. }}$.

\section{APD testing}

Initial tests were carried out on a commercially available thick junction APD (EG \& G, C30902E) [8]. The form of quenching we are using at this stage is passive quenching. We have chosen this as it allows a simple yet accurate view of the actual performance characteristics of the APD, without having to be concerned overly with the complex operating characteristics of an active circuit design. The APD characteristics (like junction capacitance, etc) have a large effect on the performance of the circuit as a whole, and as such we are concerned about choosing the best available photodiode.

The parameters we have chosen to use for the passive circuit are as follows. The load resistance is $47 \mathrm{k} \Omega$. This is a reasonably small resistance, with values usually around $200 \mathrm{k} \Omega$ being used. The smaller value allows a faster recharge time (due to reduced time constant) which allowed us to aim for testing at a $100 \mathrm{MHz}$ repetition rate. However care must be taken not to exceed the diodes maximum allowed currents and power dissipation. We used a $50 \Omega$ resistance as the matching between the APD and either a $500 \mathrm{MHz}$ digital oscilloscope or a $600 \mathrm{MHz}$ analogue oscilloscope. An approximation of the resulting waveform can be seen in Fig. 5. The C30902E was rated for a 0.5 ns rise time, so the loading effect of the resistance can clearly be seen.
The rated rise time of this APD (the C30902E) is not sufficient to meet the $3 \mathrm{ps}$ time resolution requirement. This photodiode is a thick junction APD, which has a slower rise time than the thin junction APDs. It is clear from these tests that commercially available thick junction APDs cannot be quenched in the required time and future development must therefore seek to customize faster thin junction APDs.

\section{Conclusion}

The advances that have been made in optoelectronic components and laser sources have opened up new possibilities for a wide variety of applications. Femtosecond lasers have enabled very short light bursts to be created, and with optoelectronic detectors heavily used in telecommunications, detectors capable of resolving these pulses are within reach. APDs as the front end of these detectors allow applications that depend on single photon counting to be implemented. The internal avalanche gain allows the photon arrival event to be closely mirrored with a current pulse.

The detection of the resultant current pulse is a difficult task in itself. Two forms of circuits, passive and active, are available with their relative merits. The simplicity of the passive circuit is offset by the speed advantages offered by the active circuitry, at the cost of design complexity. With our aims of a 3 ps resolution time, it is very important that the quenching circuit does not load the APD and cause the detection times to increase. The APDs cause problems of their own. Due to the high voltages and currents involved carriers can become trapped in the junction that must be counteracted by the designer by introducing a dead time, which in turn reduces the repetition rate.

A resolution time of 3 ps would enable applications like imaging of cancers in the human breast. This technique would complement the existing mammogram by enabling the detection of diffuse tumors that are currently difficult to 
detect using the mammogram. This time resolution allows a spatial detection distance of $1 \mathrm{~mm}$ in a $50 \mathrm{~mm}$ tissue sample.

Further work in the field of time resolved single photon counting for use in medical imaging could lead to improved circuit performance for even better resolution. As the relevant technology becomes smaller, faster and cheaper, this medical imaging technique could easily become used throughout industry as a complement to the traditional X-ray. As mentioned before, other applications that can potentially use this technology include astronomical observation, optical range finding, and optical fiber testing and fault location.

\section{References}

[1] S. Cova, A. Lacaita, C. Samori, M. Ghioni, Performance Optimisation of active quenching circuits for picosecond timing with single photon avalanche diodes, Review of Scientific Instruments 66 (8) (1995) 4289-4295.

[2] S. Cova, A. Lacaita, C. Samori, M. Ghioni, F. Zappa, Avalanche Photodiodes and quenching circuits for single photon detection, Applied Optics 35 (12) (1996) 1956-1976.

[3] C. Samori, M. Ghioni, S. Cova, Compact active quenching circuit for fast photon counting with avalanche photodiodes, Review of Scientific Instruments 67 (10) (1996) 3440-3448.

[4] W.J. Kindt, N.H. Shahrjerdy, H.W. van Zeijl, A silicon avalanche photodiode for single optical photon counting in the Geiger mode, Sensors and Actuators A 60 (1997) 98-102.

[5] S. Cova, M. Ghioni, A. Spinelli, L. Davis, Avalanche detector with ultraclean response for time-resolved photon counting, IEEE Journal of Quantum Electronics 34 (5) (1998) 817-821.

[6] T.P. Grayson, L.J. Wang, 400 picosecond time resolution with a passively quenched APD, Applied Optics 32 (16) (1993) 2907-2910.

[7] S. Vasile, P. Gothoskar, D. Sdrulla, R. Farrell, Photon Detection with high gain avalanche photodiode arrays, IEEE Transactions on Nuclear Science 45 (1998) 720.

[8] Device nos C30902S, C30902S-TC, C30902S-DTC, EG \& G Optoelectronics APD data sheets, 1999. 